friction coefficients, are provided in Fig. 6(a) and (b) for the three types of loading. The experimental curve for finite friction is shown along with the corresponding analytical curves for ideally smooth $(\mu=0)$ and ideally rough $(\mu \rightarrow \infty)$ interfaces. The trends of the experimental load-separation relations appear to be satisfactory as these data for $\mu=0.2$ lies between the analytical curves for $\mu=0$ and $\mu \rightarrow \infty$.

At small loads closer to the initiation of separation, the experimental curve was closer to the ideally smooth interface curve and, at higher loads, the tendency was to get closer to the ideally rough interface curve. Slip initiated at loads much smaller than the separation loads and progressed quite rapidly. For the friction coefficient $\mu=$ 0.2 ) of the models the slip spread over considerable extent by the time the separation initiated and the situation was close to smooth interfacial conditions. With increasing load, the separation progressed and the slipping process was decelerated. This process was physically equivalent to an increasing friction coefficient. Thus, gradually the load vs. separation curve with finite friction shifted to ideally rough $(\mu \rightarrow \infty)$ interface curve. Similar trends were also seen from the stress plots.

\section{Maximum Shear Stress along the Edge of the Hole}

The measurements for maximum shear stress $\tau_{\max }$ are recorded at a few important locations along the hole boundary (at $\theta=0 \mathrm{deg}, 90 \mathrm{deg}$ for sheet-loading cases). The variations of $\tau_{\max }$ vs. load are shown in Figs. 7(a), (b) and (c) for sheet loading $S_{x}$ and pin loading $P_{x}$, Case 1 and Case 2. From Figs. 7(a) and (b), it is seen that the present experimental curve lay between the two limiting cases of ideally smooth $(\mu=0)$ and ideally rough $(\mu \rightarrow \infty)$ interfacial conditions. This variation corresponaed to that due to finite interfacial friction. The $\tau_{\max }$ values for pin loading, Case 2, are seen in Fig. 7(c) and it is seen that the stress values for this case are much smaller than those due to pin load, Case 1. The consistent progress of increasing fringe order with increasing progress of separation for all the loading cases is shown in Fig. 8(a), (b) and (c).

\section{Conclusion}

In this paper, an experimental procedure was presented to obtain load-contact relationship in the presence of finite interfacial friction. While for the sheet-load case, width of sheet-to-hole ratio played a significant part in determining stress concentration and load-contact relation, it would appear that length of the plate to hole ratio played an important role in determining load-contact relationship for the pin load, Case 1 .

\section{References}

1. Frocht, M.M. and Hill, H.N., "Stress Concentration Factors around a Central Circular Hole in a Plate Loaded through Pin in a Hole," Trans. ASME, J. Appl. Mech., 62, A5-A9 (March 1940).

2. Jessop, H.T., Snell, C. and Hollister, G.S., "Photoelastic Investigation on Plates with Single Interference Fit Pins with Load Applied to Plate Only,"' Aeronaut. Quart., 7, 297-315 (1956).

3. Jessop, H.T., Snell, C. and Hollister, G.S., "Photoelastic Investigations on Plates with Single Interference Fit Pins with Load Applied to (a) Pin Only and (b) Pin and Plate Simultaneously," Aeronaut. Quart., 9, 147-163 (1958).

4. Lambert, T.H. and Brailey, R.J., "The Influence of the Coefficient of Friction on the Elastic Stress Concentration Factor for a Pin Jointed Connection,"'Aeronaut. Quart., 13, 17-29 (1962).

5. Cox, H.L. and Brown, A.F.C., "Stresses Round Pins in Holes," Aeronaut. Quart., 15, 357-372 (1964).

6. ESDU Engineering Science Data Unit, 251-259, Regent Street, London, WIR 7AD, England.

7. Rao, A.K., "Accurate Determination of Interference in Pin Joints, "'EXPERIMENTAL MECHANICS, 9 (2), 45-48 (1969).

8. Venkataraman, N.S., "A Study into the Analysis of Interference Fits and Related Problems," PhD Thesis, Indian Institute of Science, Bangalore, India (1966).

9. Eshwar, V.A., "Partial Contact and Friction in Pin Joints," PhD Thesis, Indian Institute of Science, Bangalore, India (Dec. 1977).

10. Ghosh, S.P., "Analysis of Joints with Elastic Pins," PhD Thesis, Indian Institute of Science, Bangalore, India (Nov. 1978).

11. Rao, A.K., "Elastic Analysis of Pin Joints," Invited Paper in Int. Jour. of Comp. and Strucs., Special issue commemorating the 65th birthday of Prof. J.H. Argyris of the Univ. of Stuttgart and the Imperial College of Sci, and Tech; Published in 9 (2) (1978).

I2. Durelli, A.J. and Riley, R.J., "Introduction to Photomechanics," Prentice-Hall (1965).

\title{
Errata and Addendum
}

"Strain-rate History and Temperature Effects on the Torsional-shear Behavior of a Mild Steel" by Abdel-Salam M. Eleiche

An error in the original preparation of the text has been discovered by the Author. On page 291 of the August 1981 issue of EXPERIMENTAL MECHANICS, eq (5), giving an appropriate constitutive relation for the mild steel tested under constant-strain-rate conditions, should read:

$$
\tau=K \gamma^{n}\left(\tau_{o}+\mu_{12} \ell \mathrm{n} \dot{\gamma}\right) \exp \left(-B T_{a}\right)
$$

where $B, K, n, \tau_{0}$ and $\mu_{12}$ are material constants, functions of strain, strain rate and temperature, and whose values for the present test conditions are given in Tables 1, 2, 3 and 4.
TABLE 4-VALUES OF $K$, FOR VARIOUS TEMPERATURES $T_{a}$, STRAINS $\gamma$, AND STRAIN RATES $\dot{\gamma}$ IN THE EQUATION $\tau=K \gamma^{n}\left(\tau_{o}+\mu_{12} \ell \mathrm{n} \dot{\gamma}\right) \exp \left(-B T_{\alpha}\right)$

\begin{tabular}{|c|c|c|c|c|c|c|c|c|}
\hline$\dot{\gamma}\left(s^{-1}\right)$ & \multicolumn{4}{|c|}{0.006} & \multicolumn{4}{|c|}{1000} \\
\hline$|\boldsymbol{a}|$ & 0.05 & 0.10 & 0.25 & 0.50 & 0.05 & 0.10 & 0.25 & 0.50 \\
\hline 123 & 2.30 & 1.96 & 1.64 & 1.49 & 1.92 & 1.63 & 1.47 & 1.34 \\
\hline 173 & 3.19 & 2.54 & 2.00 & 1.74 & 2.08 & 1.87 & 1.65 & 1.49 \\
\hline 223 & 4.54 & 3.38 & 2.46 & 2.05 & 2.43 & 2.15 & 1.86 & 1.65 \\
\hline 293 & 6.43 & 4.50 & 3.08 & 2.50 & 3.07 & 2.65 & 2.22 & 1.91 \\
\hline 473 & 10.58 & 6.89 & 4.57 & 3.77 & 5.60 & 4.49 & 3.47 & 2.78 \\
\hline 673 & 29.63 & 16.00 & 8.81 & 6.66 & 8.95 & 6.95 & 5.20 & 4.04 \\
\hline
\end{tabular}

